\title{
Accelerated decolorization of azo dye Congo red in a combined bioanode-biocathode bioelectrochemical system with modified electrodes deployment
}

\author{
Fanying Kong ${ }^{\mathrm{a}}$, Aijie Wang ${ }^{\mathrm{a}, *}$, Haoyi Cheng ${ }^{\mathrm{b}}$, Bin Liang ${ }^{\mathrm{a}}$ \\ ${ }^{a}$ State Key Laboratory of Urban Water Resource and Environment, School of Municipal and Environmental Engineering, Harbin Institute of Technology, Harbin 150090, China \\ ${ }^{\mathrm{b}}$ Research Center for Eco-Environmental Sciences, Chinese Academy of Sciences, Beijing 100085, China
}

\section{H I G H L I G H T S}

- CR decolorization was accelerated with combined bioanode-biocathode BES.

- The biocathode played an important role in accelerating CR decolorization.

- Three electrodes deployments were compared based on startup, DE and EIS analysis.

- The surrounding mode was the best, followed by vertical and horizontal ones.

- The possible mechanism for the combined bioanode-biocathode was discussed.

\section{A R T I C L E I N F O}

\section{Article history:}

Received 2 September 2013

Received in revised form 3 October 2013

Accepted 9 October 2013

Available online 21 October 2013

\section{Keywords:}

Bioelectrochemical system (BES)

Azo dye Congo red (CR)

Decolorization efficiency (DE)

Biocathode

Bioanode

\begin{abstract}
A B S T R A C T
In this study, BES with bioanode and biocathode was applied to decolorize an azo dye Congo red (CR). Results showed that the Congo red decolorization efficiency (CR-DE) within $23 \mathrm{~h}$ in a combined bioanode-biocathode single chamber BES was $98.3 \pm 1.3 \%$, significantly higher than that of mixed solution in a dual chamber BES $(67.2 \pm 3.5 \%)(P<0.005)$. Various electrodes deployments (horizontal, vertical and surrounding) in the combined bioanode-biocathode BES were further compared based on the decolorization performance and electrochemical characterization. Results indicated that CR-DE within $11 \mathrm{~h}$ improved from $87.4 \pm 1.3 \%$ to $97.5 \pm 2.3 \%$, meanwhile the internal resistance decreased from 236.6 to $42.2 \Omega$ as modifying the horizontal deployment to be a surrounding deployment. It proved that the combination of bioanode and biocathode with suitable electrodes deployment could accelerate the decolorization of azo dye Congo red, which would be great potential for the application of bioelectrochemical technology in azo dye wastewater treatment.
\end{abstract}

(C) 2013 Elsevier Ltd. All rights reserved.

\section{Introduction}

Bioelectrochemical technology has recently drawn increasing attention in azo dyes treatment (Fernando et al., 2012; Hou et al., 2012; Luo et al., 2011; Mu et al., 2009; Solanki et al., 2013; Wang et al., 2013a,b). There are two modes for azo dyes decolorization in BES. One is cathode decolorization in a dual chamber BES: Substrate is oxidized by bacteria at anode to produce protons and electrons, which are transferred to the cathode via membrane and external circuit, respectively. At the cathode, the azo bonds of dye are broken using proton and electron, resulting in the formation of colorless products (Mu et al., 2009; Solanki et al., 2013). In this mode, azo dye decolorization is mainly attributed to the electrochemical reduction for the absence of microorganism (Mu

\footnotetext{
* Corresponding author. Tel./fax: +86 45186282195.

E-mail address: waj0578@hit.edu.cn (A. Wang).
}

et al., 2009). Few papers focused on the azo dye decolorization with dual microbial or enzymatic cathode BES (Cui et al., 2011; Savizi et al., 2012). The other is anode decolorization in an aircathode single chamber BES: Azo dye is readily transformed by anode microorganisms through reductive cleavage of azo bond to form aromatic amines in the presence of co-substrate. Due to the fact that electrons produced from substrate were transferred to the cathode for oxygen reduction rather than azo dye reduction for the higher oxygen redox potential (Cao et al., 2010; Hou et al., 2011a,b). The co-metabolism-dependent bioreduction is mainly responsible for the decolorization while less electrochemical reduction does (Cao et al., 2010).

The biocathode is proving to be a promising feature for development of BES. It has been reported that the reduction reactions at cathode especially biocathode can enhance wastewater treatment, such as pentachlorophenol, hexavalent chromium, nitrobenzene and chlorinated nitroaromatic antibiotic chloramphenicol etc. 

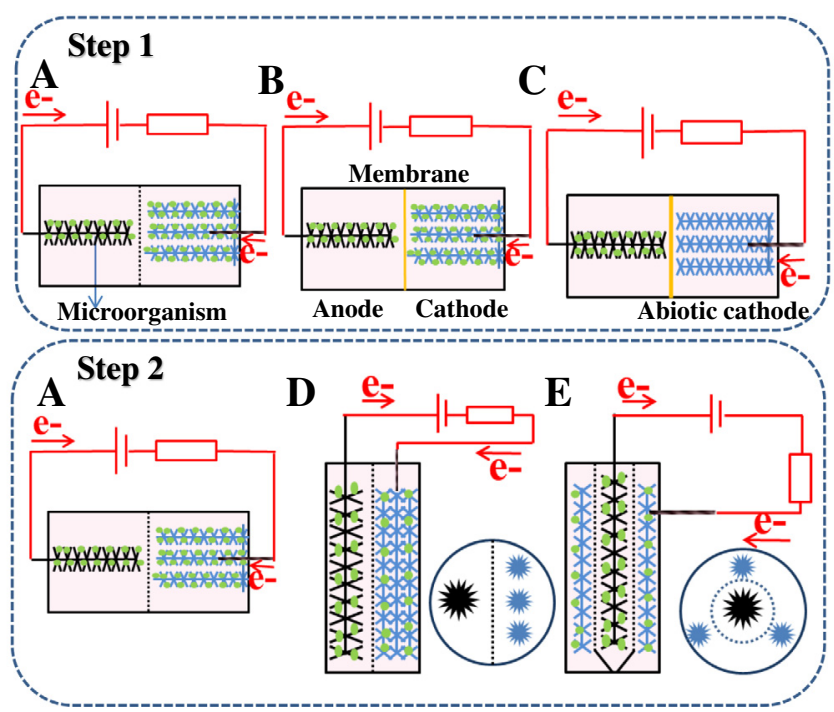

Fig. 1. Bioelectrochemical reactor configurations and operation. (A) Single chamber BES with combined bioanode-biocathode (horizontal electrodes deployment); (B) dual chamber BES with bioanode and biocathode; (C) dual chamber BES with bioanode and abiotic cathode; (D) single chamber BES with combined bioanodebiocathode (vertical electrodes deployment); (E) single chamber BES with combined bioanode-biocathode (surrounding electrodes deployment).

Table 1

The main characteristics of the activated sludge.

\begin{tabular}{ll}
\hline Parameter & Value \\
\hline $\mathrm{pH}$ & 6.73 \\
TSS (total suspended solids) & $2.26 \mathrm{~g} \mathrm{~L}^{-1}$ \\
VSS (volatile suspended solids) & $1.40 \mathrm{~g} \mathrm{~L}^{-1}$ \\
TCOD (total chemical oxygen demand) & $1.84 \mathrm{~g} \mathrm{~L}^{-1}$ \\
SCOD (soluble chemical oxygen demand) & $42.70 \mathrm{mg} \mathrm{L}^{-1}$ \\
Solute protein (as COD) & $12.88 \mathrm{mg} \mathrm{L}^{-1}$ \\
Solute carbohydrate (as COD) & $1.47 \mathrm{mg} \mathrm{L}^{-1}$ \\
\hline
\end{tabular}

(Aulenta et al., 2011; Huang et al., 2011, 2013; Liang et al., 2013; Wang et al., 2011; Xafenias et al., 2013), gaining more and more interest as it accelerates reduction reactions and circumvents the utilization of artificially added mediators, however, less literature refers to azo dyes decolorization with biocathode BES (Cui et al., 2011; Wang et al., 2013a). Thus, it is necessary to investigate the decolorization performance of azo dyes using biocathode BES. Furthermore, there are some studies on combination of anode and cathode in BES for wastewater or groundwater treatment ( $\mathrm{Li}$ et al., 2010; Lohner et al., 2011; Sun et al., 2011a; Wen et al., 2010). Considering the bioanode decolorization at present, air- cathode single chamber MFC has been used extensively (Cao et al., 2010; Hou et al., 2011b, 2012; Xu et al., 2013). The air-cathode was chosen for minimizing the effect from dye and cathode (Cao et al., 2010) but was limited by the unavailable cathode reduction role in the decolorization process. This will make no effective use of the whole BES reactor in wastewater treatment. If the feasibility of biocathode decolorization is further verified, the development of a combined bioanode-biocathode system will become a promising technology for enhanced azo dye decolorization.

Present BES reactor configurations for decolorization vary from rectangular to tubular and to sleeve-type (Cui et al., 2011, 2012; Kong et al., 2013). It has been demonstrated that a suitable BES structure will be a necessary consideration for large-scale MFC design, including electrode surface area and electrode spacing (Liu et al., 2008; Zhang et al., 2013b). However, it is inevitable to consider the key design factors when the number of electrodes increases to a certain amount in the scale-up process (Zhu et al., 2013). Previous work in the laboratory has demonstrated the feasibility of reducing internal resistance and enhancing decolorization performance in a modified sleeve-type dual chamber BES for azo dye acid orange decolorization (Kong et al., 2013). If modifying the electrodes deployment of single chamber BES to be sleevetype, namely the cathode electrodes surrounding the anode electrode, it may also facilitate electron transfer and increase the effective area of anode and cathode, further improving azo dye decolorization performance in the combination of bioanode and biocathode BES. Although different reactor structures have been used during the last years, there is no systematic information regarding the influence of electrodes deployment on decolorization and electrochemical characterization in the single chamber BES.

Based on the above consideration, this study aims to develop a single chamber BES with a combination of bioanode and biocathode, and make full use of bioanode and biocathode in azo dye decolorization. Further focus on the comparison of biocathode and abiotic cathode was also studied to confirm the important role of microbial in azo dye decolorization at biocathode. Three different electrodes deployments (horizontal, vertical and surrounding) were compared based on the azo dye decolorization performance and electrochemical catalytic characterization to determine the optimum electrodes deployment in single chamber BES.

\section{Methods}

\subsection{Configurations of bioelectrochemical system}

The single chamber reactor was constructed by a cylindrical Plexiglas tube (ID $8 \mathrm{~cm} \times \mathrm{H} 10 \mathrm{~cm}$ ) without membrane (Fig. 1A, D and E). The dual chamber reactor with an anode chamber and a cathode chamber separated by a cation exchange membrane

Table 2

Experimental setup.

\begin{tabular}{|c|c|c|c|c|c|}
\hline No. & Purpose & Reactor & Chamber & Electrolyte & Time $(h)$ \\
\hline \multirow[t]{2}{*}{1} & \multirow{2}{*}{$\begin{array}{l}\text { Performance in combined bioanode-biocathode BES } \\
\text { and separated bioanode and biocathode BES }\end{array}$} & Combined bioanode and biocathode (Fig. 1A) & Single & $\mathrm{CR}+$ glucose & 23 \\
\hline & & Separated bioanode and biocathode (Fig. 1B) & Dual & $\begin{array}{l}\text { Anolyte: } C R+\text { glucose } \\
\text { Catholyte: CR + glucose }\end{array}$ & 23 \\
\hline \multirow[t]{3}{*}{2} & \multirow[t]{3}{*}{ Microbial role at biocathode } & Biocathode (Fig. 1B) & Dual & $\begin{array}{l}\text { Anolyte: } C R+\text { glucose } \\
\text { Catholyte: CR + glucose }\end{array}$ & 23 \\
\hline & & Biocathode (Fig. 1B) & Dual & $\begin{array}{l}\text { Anolyte: } C R+\text { glucose } \\
\text { Catholyte: } C R\end{array}$ & 23 \\
\hline & & Abiotic cathode (Fig. 1C) & Dual & $\begin{array}{l}\text { Anolyte: } C R+\text { glucose } \\
\text { Catholyte: } C R\end{array}$ & 23 \\
\hline \multirow[t]{3}{*}{3} & \multirow[t]{3}{*}{ Effect of electrodes deployments } & Horizontal (Fig. 1A) & Single & $\mathrm{CR}+$ glucose & 23 \\
\hline & & Vertical (Fig. 1D) & Single & $\mathrm{CR}+$ glucose & 23 \\
\hline & & Surrounding (Fig. 1E) & Single & $\mathrm{CR}+$ glucose & 23 \\
\hline
\end{tabular}



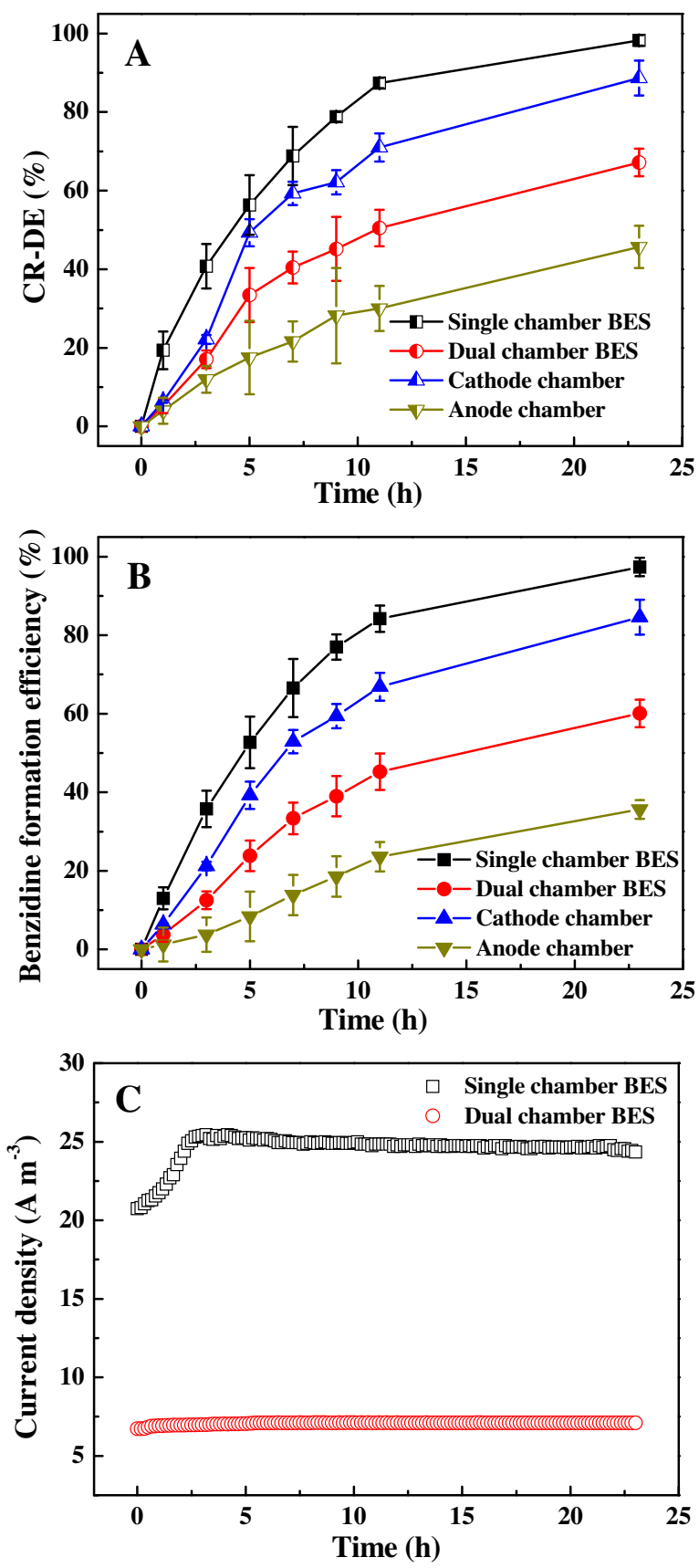

Fig. 2. Congo red decolorization (A), benzidine formation (B) and current density (C) in combined bioanode-biocathode single chamber BES and in separated bioanode/biocathode dual chamber BES.

(CEM, Ultrex CMI7000, Membranes International Inc., USA) was used as the control (Fig. 1B and C). In all the tested single chamber reactors, the total working volume was $200 \mathrm{~mL}$ plus a certain headspace, while that of rectangular dual chamber reactor was $100 \mathrm{~mL}$ for anode chamber and $100 \mathrm{~mL}$ for cathode chamber. The systems were sealed carefully to maintain anaerobic condition. Both anode and cathode electrodes were carbon brush (ID $3 \mathrm{~cm} \times \mathrm{L} 6 \mathrm{~cm}$ ) made of carbon fibers (Jilin Carbon Plant, China). The deployments of electrodes were exhibited as Fig. 1, with anode and cathode ratio of 1:3. Three electrodes deployments were tested in this study, including horizontal deployment (Fig. 1A), vertical deployment (Fig. 1D) and surrounding deployment (Fig. 1E). The anode, cathode and the reference electrode (saturated calomel reference electrode, SCE, model-217, Shanghai Precise. Sci. Instru. Co., Ltd., China;
$0.247 \mathrm{~V}$ vs. standard hydrogen electrode, SHE) were connected to a data acquisition (Keithley 2700, Keithley Co., Ltd., USA) with external resistance of $20 \Omega$.

\subsection{Operation conditions}

Activated sludge obtained from Taiping wastewater treatment plant (Harbin, China) was used as the inoculum of the anode and cathode $(10 \% \mathrm{~V} / \mathrm{V})$. The main characteristics of activated sludge were displayed in Table 1. The BES reactors (except as noted) were fed a solution containing Congo red (model of azo dye, $300 \mathrm{mg} \mathrm{L}^{-1}$ ), glucose $\left(500 \mathrm{mg} \mathrm{L}^{-1}\right)$ in a medium as described previously (Xing et al., 2009). Before each batch cycle, $\mathrm{N}_{2}$ gas was flushed continuously for 15 min to maintain anaerobic conditions.

The experiments were performed with applied voltage of $0.3 \mathrm{~V}$. According to the peak current from $\mathrm{CV}$, the cathode potential for $\mathrm{CR}$ decolorization was $-0.7 \mathrm{~V}$ (Fig. 4A). Only with the applied voltage of $0.3 \mathrm{~V}$, the cathode potential could be kept at $-0.7 \mathrm{~V}$ as the anode potential was stable at $-0.4 \mathrm{~V}$ or even lower. Comparison of combined bioanode-biocathode in the single chamber BES with separated bioanode and biocathode in dual chamber BES was investigated (Fig. 1A and B). In order to further understand the biocathode role in azo dye decolorization, biocathode with or without glucose (Fig. 1B) was also compared with abiotic cathode without glucose (Fig. 1C) in the dual chamber BES. Moreover, BES reactors with different electrodes deployments were employed in this study, including horizontal deployment (Fig. 1A), vertical deployment (Fig. 1D) and surrounding deployment (Fig. 1E), to further investigate the effect of electrodes deployment on the CR decolorization and electrochemical characterization. Detail information in experimental setup was displayed in Table 2. Performance of each reactor under biocatalyzed electrolysis system (BES), microbial fuel cell (MFC) and anaerobic bioreactor (AnBR) was also studied. In addition, the BES was the bioelectrochemical reactor applied a voltage of $0.3 \mathrm{~V}$ using a power supply. The MFC was bioelectrochemical reactor disconnected of additional power supply. The anaerobic bioreactor (AnBR) was the open circuit bioreactor.

\subsection{Analytical methods}

The CR concentration was measured by UV-visible scanning spectrophotometer (Shimadzu UV2550, Japan) at $\lambda_{\max } 496 \mathrm{~nm}$ (Hou et al., 2011b). Samples were centrifuged at $10,000 \mathrm{rpm}$ for 10 min to remove suspended biomass from the liquid media before absorbance was measured. After filtering the samples through a $0.45 \mu \mathrm{m}$ filter, the decolorization products were analyzed using high performance liquid chromatography (HPLC, model e2695, Waters Co., USA) with a C18 column $(5 \mu \mathrm{m} ; 5 \mathrm{~mm} \times 250 \mathrm{~mm}$, Waters Co., USA). It performed with methanol $/ \mathrm{H}_{2} \mathrm{O}(55: 45 ; \mathrm{v} / \mathrm{v})$ as the mobile phase at a flow rate of $0.6 \mathrm{~mL} \mathrm{~min}^{-1}$ with UV detection at $270 \mathrm{~nm}$. CR decolorization efficiency (CR-DE) was calculated according to the previous report (Cao et al., 2010). The kinetic of CR decolorization was modeled using first order kinetic model $C=C_{0}$ $\mathrm{e}^{-k t}$, where $C$ is $C R$ concentration $\left(\mathrm{mg} \mathrm{L}^{-1}\right)$ at time $t(\mathrm{~h})$, and $C_{0}$ is the initial $C R$ concentration $\left(\mathrm{mg} \mathrm{L}^{-1}\right)$. Statistical significance of differences was analyzed by two-tailed unpaired $t$-test.

Anode and cathode as well as the reference electrode were connected to a data acquisition unit (Keithley 2700, Keithley Co., Ltd., USA) to record the potentials and current every $10 \mathrm{~min}$. The current density was calculated based on the reaction liquid volume. Coulombic efficiency (CE) was evaluated by benzidine formation ( $\left.\mathrm{CE}_{\text {benzidine }}\right)$, which was calculated as the ratio of the theoretical coulombs based on the benzidine formation ( $8 \mathrm{~mol}$ electrons per mol benzidine) and the coulombs calculated by integrating the current over time (Mu et al., 2009). Cyclic voltammetry (CV) was measured with the scan rate of $5 \mathrm{mV} \mathrm{s}^{-1}$ using an electrochemical 

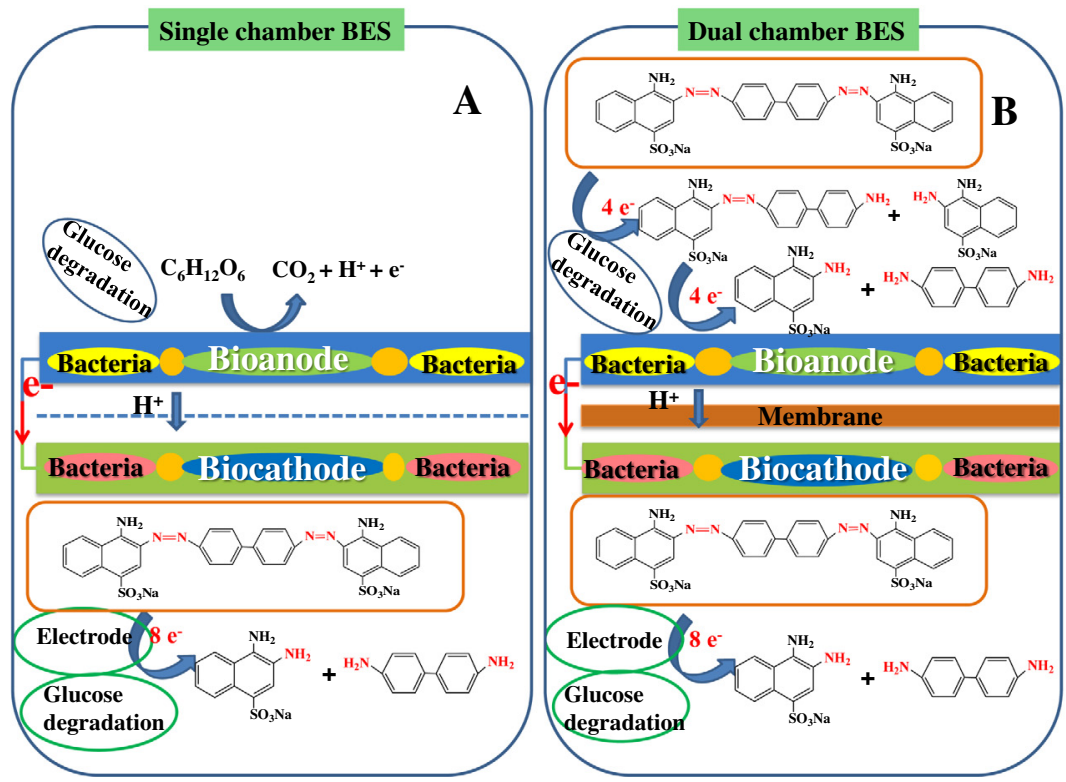

Fig. 3. The possible mechanism with combined bioanode-biocathode in single chamber BES (A) and separated bioanode/biocathode in dual chamber BES (B).

workstation (model-660D, CH Instruments Inc., USA) equipped with three-electrode system (cathode as working electrode, anode as counter electrode and SCE as reference electrode). Electrochemistry impedance spectroscopy (EIS) measurements were carried in a frequency range of $0.01-10^{5} \mathrm{~Hz}$ at open circuit condition with a perturbation signal of $10 \mathrm{mV}$ (Kong et al., 2013).

\section{Results and discussion}

\subsection{Enhanced performance with combined bioanode and biocathode}

Considering that the $\mathrm{CR}$ decolorization at bioanode has been reported in air-cathode single chamber BES in the previous researches (Hou et al., 2011a; Sun et al., 2011b, 2012, 2013), this study aimed to associate biocathode with bioanode to realize the improved decolorization of CR. Two different modes were studied for comparison, including the combined bioanode-biocathode in a single chamber BES and the separated bioanode/biocathode in a dual chamber BES.

Results showed that CR-DE within $23 \mathrm{~h}$ in the combined bioanode-biocathode single chamber BES $(98.3 \pm 1.3 \%)$ was about $30 \%$ higher than that of mixed solution in the dual chamber BES obtained from anode chamber and cathode chamber $(67.2 \pm 3.5 \%$, $P<0.005$ ) with $300 \mathrm{mg} \mathrm{L}^{-1} \mathrm{CR}$ and $500 \mathrm{mg} \mathrm{L}^{-1}$ glucose. In addition, the CR-DE in the separated biocathode and bioanode mode was significantly decreased to $88.7 \pm 4.4 \%(P<0.05)$ and $45.5 \pm 5.2 \%$ $(P<0.005)$, respectively (Fig. $2 A)$. The current density of single chamber BES was about $25.1 \mathrm{~A} \mathrm{~m}^{-3}$, which was 3.6-fold higher than that of dual chamber BES $\left(7.1 \mathrm{~A} \mathrm{~m}^{-3}\right.$ ) (Fig. 2C). It strongly indicated that the anode performance was the limiting factor on the whole CR-DE. The bioanode in the dual chamber was negatively affected by $C R$ which might have toxicity effect on anode microorganisms, restricting the oxidation of glucose or reduction of $\mathrm{CR}$ in anode chamber, and indirectly restraining $\mathrm{CR}$ decolorization in the cathode chamber due to the electrons generated from anode. This might finally resulted in the relative low CR-DE both at anode and cathode. However, in the single chamber BES with both biocathode and bioanode in a reactor, the bioanode might be mainly used to oxidize glucose to provide electrons for $\mathrm{CR}$ reduction, while the cathode might be mainly used for the $C R$ decolorization, leading to the less toxicity effect on microbes and resulting in the better decolorization performance. Coulombic efficiency based on the product benzidine ( $\left.\mathrm{CE}_{\text {benzidine }}\right)$ was calculated as $29.2 \%$ and $41.4 \%$ in single chamber BES and dual chamber $B E S$, respectively. The coulombs loss (due to $C E<100 \%$ ) might be caused by the parallel reductive reactions occurred on cathode, such as hydrogen evolution, due to the low cathode potential here ( $\sim-800 \mathrm{mV}$ vs. $\mathrm{Ag} / \mathrm{AgCl})$.

The possible mechanism of CR degradation in the single chamber BES with combined bioanode-biocathode and dual chamber BES with separated bioanode/biocathode was proposed in Fig. 3. Azo dye, which is aromatic compounds with one or more $-\mathrm{N}=\mathrm{N}-$ groups, could be decolorized with cleavage of azo bonds. In the single chamber BES, the bioanode might play a significant role in the glucose oxidation to generate electrons while the biocathode might be responsible for reductive cleavage of azo bonds. Electrons from glucose oxidation at bioanode and/or biocathode might be also captured by anodophilic and/or cathodophilic microbes for $\mathrm{CR}$ reduction (Fig. $3 \mathrm{~A}$ ). In the dual chamber BES, glucose was oxidized by electrochemical active microorganisms at anode. The electrons released from glucose oxidation could work as additional electrons for $\mathrm{CR}$ reduction (co-metabolism process) in the anode chamber. Meanwhile partial electrons transported to the cathode through an electrical circuit for reductive cleavage of azo bonds in the cathode chamber, resulting in the formation of aromatic amines. Except of the electrons from electrode, the glucose in the cathode chamber might also act as additional electrons donor for CR decolorization (Fig. 3B).

The samples were further analyzed by HPLC to identify the reduced products of the $\mathrm{CR}$ decolorization based on the standard samples. According to the possible mechanism, the same possible product of CR reduction in the separated chamber BES and dual chamber BES was benzidine, thus the formation of benzidine was compared specially. As shown in Fig. 2B, benzidine was also produced rapidly in combined bioanode and biocathode BES compared with that in separated ones. This transformation mechanism was consistent with that in the air-cathode single chamber BES, however, it was different from that the destruction of the chromogenic group $\left(-\mathrm{NH}_{2}\right)$ on aromatic rings was observed in air-cathode BES (Sun et al., 2013), possibly explaining by the micro-aerobic environment near the air-cathode. It should be noted that the effluent still need further treatment by aerobic biological process.

It can be concluded that the combined bioanode and biocathode in a single chamber BES performed better than the separated 

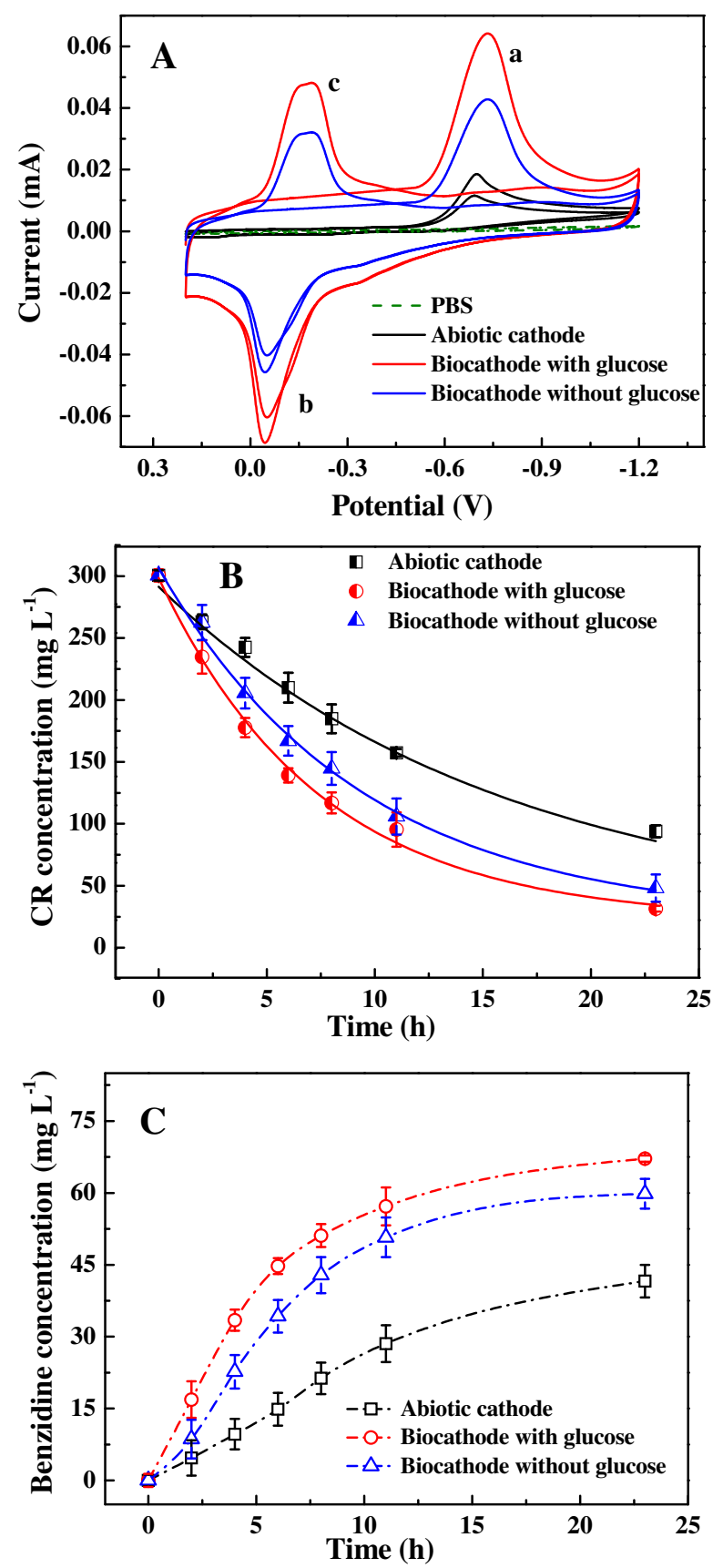

Fig. 4. Cyclic voltammograms (CV) (A), Congo red decolorization (B) and benzidine formation (C) at abiotic cathode and biocathode (with or without glucose) in the dual chamber BES.

bioanode and biocathode in a dual chamber BES, demonstrating the feasibility of using combined bioanode-biocathode to improve the decolorization of azo dye.

\subsection{Microbial role in $C R$ decolorization at biocathode}

The electrochemical activity between biocathode and abiotic cathode was investigated through CV to manifest the effect caused by cathodophilic microbes on CR reduction. The onset potential of $\mathrm{CR}$ reduction at biocathode, despite of the presence of glucose or not, was started at around $-0.5 \mathrm{~V}$, and the peak current (peak a) was obtained at about $-0.7 \mathrm{~V}$, which was similar to the abiotic cathode. While no current peak in CV was observed with the PBS as control (Fig. 4A). Therefore, the peak current observed both at biocathode and abiotic cathode was contributed by the $\mathrm{CR}$ reduction. An oxidation peak (peak b) and a new reduction peak (peak c) appeared in CV for biocathode in the next sweep cycle, accompanying with the decreased current peak (peak c) (Fig. 4A). It has been proposed that these redox peaks resulted from the reversible reaction of the intermediates (amine products) during the disruption of azo bond (Mu et al., 2009). The corresponding redox peak at abiotic cathode could be neglected compared to that of the biocathode. Thus it can be concluded that at abiotic cathode, the reaction of amine products was even small and could be neglected. The different CVs indicated that the electron transfer pathway either on biocathode or abiotic cathode might be different, which was similar to other investigations (Liang et al., 2013; Wang et al., 2011). CV results also demonstrated that the biocathode with or without glucose could efficiently catalyze $\mathrm{CR}$ reduction reaction. Much higher current at biocathode was generated than that at abiotic cathode, especially, peak current (peak a) for CR reduction
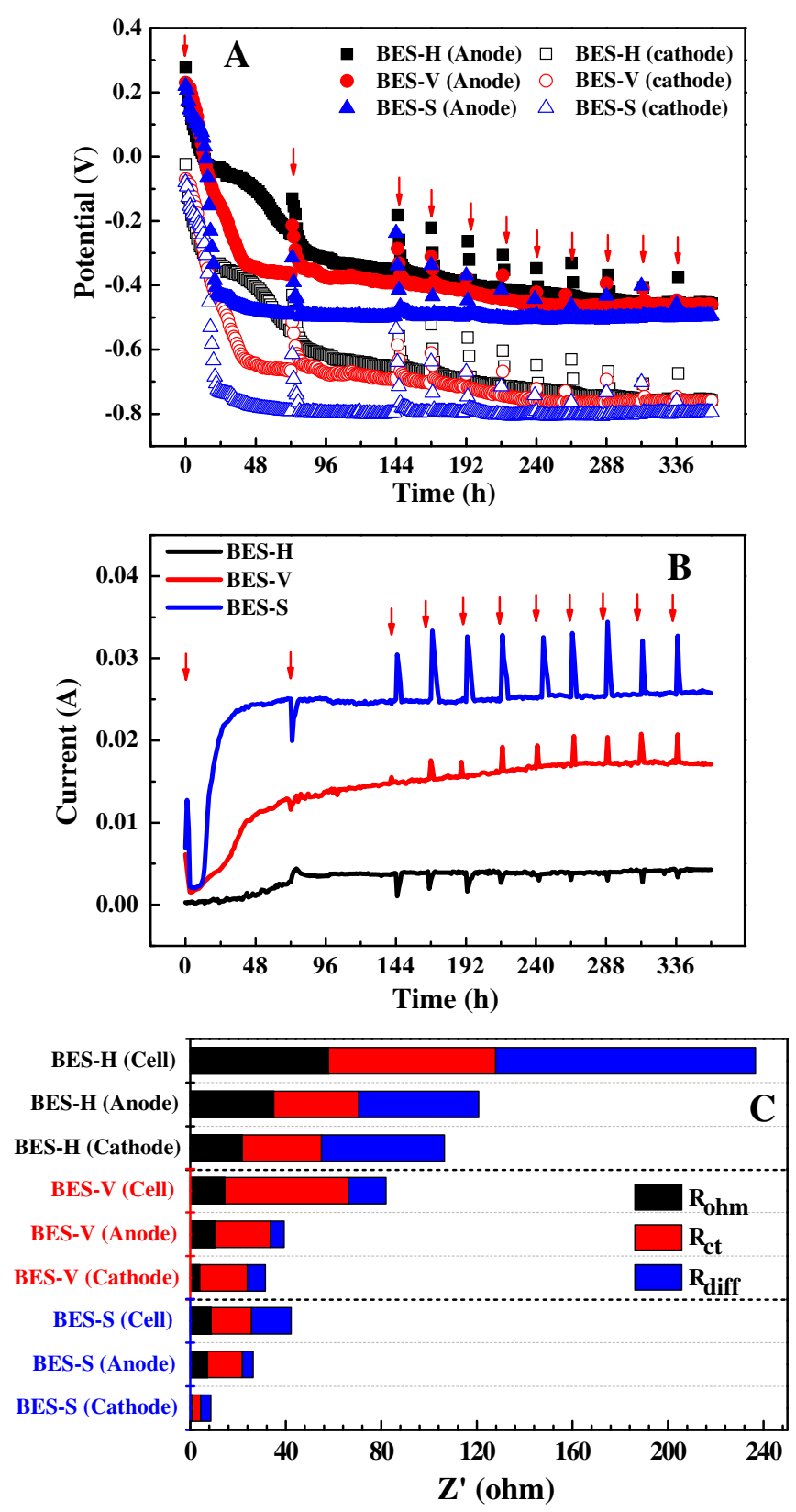

Fig. 5. Electrode potential (A), current (B) and EIS (C) with different electrodes deployments. 

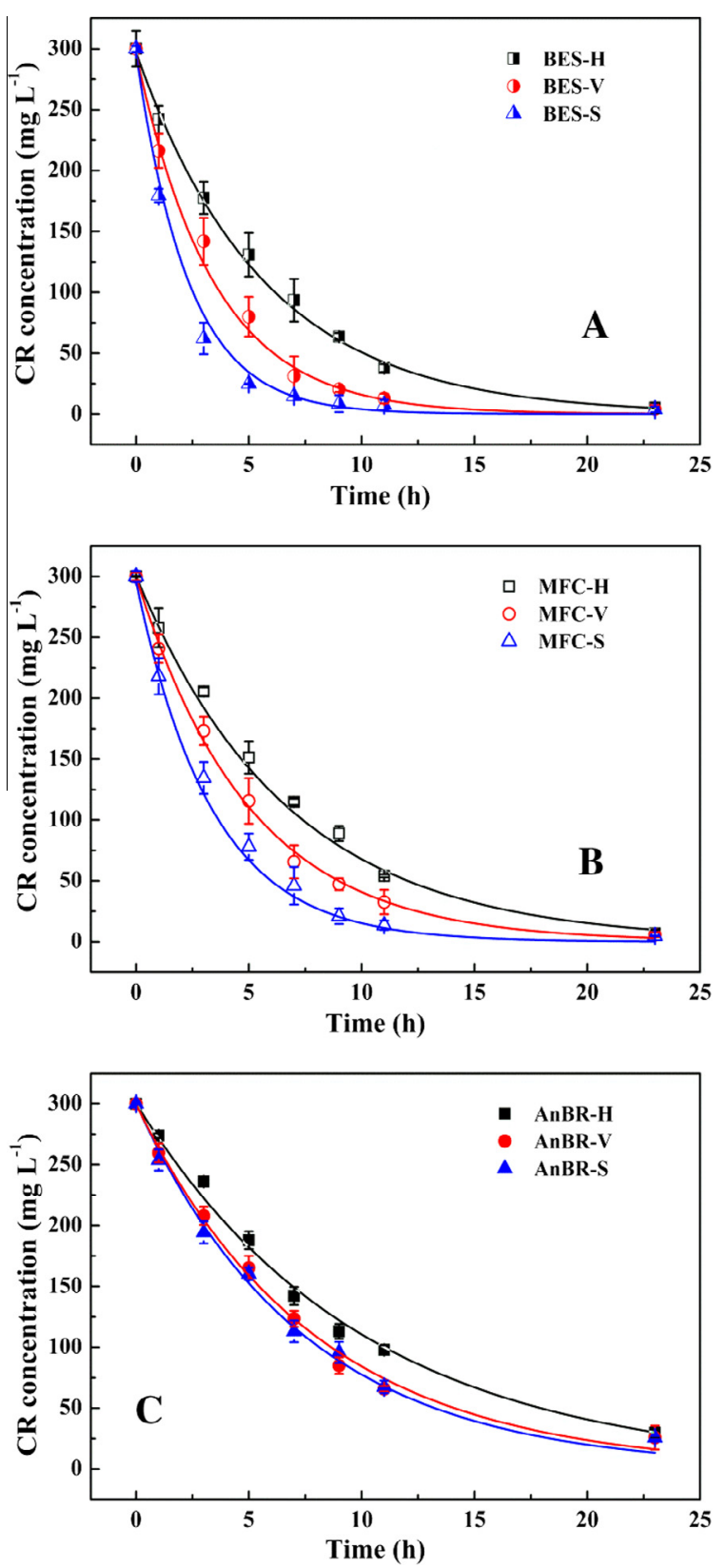

Fig. 6. The decolorization performance in different modes BES (A), MFC (B) and AnBR (C) with different electrodes deployments.

at biocathode with glucose $(0.064 \mathrm{~mA})$ or without glucose (0.043 mA) was 3.6-fold or 2.4-fold higher than that at abiotic cathode $(0.018 \mathrm{~mA})$, respectively (Fig. $4 \mathrm{~A})$. This enhanced current exhibited higher electrochemical activity for CR reduction at biocathode, indicating that the biofilm on biocathode can accelerate the $\mathrm{CR}$ bioelectrochemical reduction.

Similar result was observed in decolorization. The BES performed quite well with a biocathode with or without glucose compared with an abiotic cathode as control. The CR-DE of biocathode with glucose was $88.7 \pm 4.4 \%$ at $23 \mathrm{~h}$, while the CR-DE of abiotic cathode was $57.6 \pm 2.3 \%$, about $30 \%$ lower than biocathode $(P<0.005)$, while CR-DE of biocathode without glucose was $80.6 \pm 2.5 \%$ (Fig. 4B). The rate constant $k\left(\mathrm{~h}^{-1}\right)$ of biocathode with glucose $(0.135 \pm 0.011)$ was 2.1 times to that of abiotic cathode $(0.065 \pm 0.003)(P<0.005)$, while $k$ of biocathode without glucose $(0.107 \pm 0.008)$ was also significantly difference $(P<0.05)$.
The benzidine of CR was produced rapidly as CR reduced in the biocathode BES with or without glucose, while benzidine in the abiotic cathode BES was accumulated slowly and much lower than that in the biocathode BES. The product formation efficiency at biocathode with or without glucose was higher than $80 \%$, and that at abiotic cathode was about 52\%, further demonstrating that most of the electrons from the cathode were used for the reduction of the azo bond in CR (Fig. 4C). These indicated that the presence of biofilm at cathode did enhance the CR decolorization likely as cathodophilic microbes accelerated electrons transfer for CR reduction and the influence of glucose as additional electron donor was not obvious. Thus, it was very important to introduce biocathode into BES for azo dye decolorization, which would strengthen the bioelectrochemical decolorization process.

\subsection{Electrochemical characterization with different electrodes deployments}

After determining the better performance in the single chamber BES with combined bioanode-biocathode, it is essential to improve the key design of electrodes deployment of the BES reactor for further performance improvement. Sleeve-type has been demonstrated as an effective configuration for azo dye decolorization in the dual chamber BES (Kong et al., 2013). In this study, the electrodes deployment of single chamber BES was modified as the three cathode electrodes surrounding the anode electrode as shown in Fig. 1E. In order to better understanding the difference of horizontal and vertical electrodes deployments commonly employed in the wastewater treatment with surrounding one, the three electrodes deployments in BES reactor (BES-H, BES-V and BES-S) were compared.

The cathode potential of the three deployments started from about $-0.06 \pm 0.02 \mathrm{~V}$ after inoculation, then gradually decreased later and was stable at $-0.775 \pm 0.020 \mathrm{~V}$ even when the solution was replaced by fresh solution, indicating the success of startup. The corresponding anode potential was about $-0.475 \pm 0.020 \mathrm{~V}$ (Fig. 5A). In the contemporary BES studies, the credible steady state for the BES-S was present at $72 \mathrm{~h}$ (3 days) while that for the BES-V and BES-H was at $240 \mathrm{~h}$ (10 days) and $316 \mathrm{~h}$ (14 days) (Fig. 5A). The rapid startup might be caused by the rapid microbial cells attachment on electrodes, which would result in the rapid decrease of cathode potential and anode potential (Zhang et al., 2013c). That was to say, the most important step in biofilm formation and microbes growth could be enhanced with surrounding electrodes deployment.

The three deployments had a similar trend of an initially low current production followed by a gradually current increased phase and then stable and reproducible phase. However, the surrounding electrodes deployment in the single chamber BES could obtain better performance than vertical and horizontal ones. The BES-S had a nearly constant current of $0.025 \mathrm{~A}$ at stable stage, which was 1.47-fold higher than that observed in BES-V (0.017 A), especially 6.25-fold higher than that in BES-H (0.004 A) (Fig. 5B). Due to the fact that current generation is influenced by the transfer of protons, substrate and metabolites between electrode surface and bulk solution (Zhao et al., 2009), the different deployments may have different performance in this process, resulting in the different current. Thus, the highest current in the BES-S may be attributed to a more efficient electron transfer from microbial to electrode as BES-S had the reduced electrodes spacing and large proton exchange area, which would benefit the electron transfer and lead to a lower internal resistance and then higher current.

The EIS was measured to compare the three deployments in resistances. The I-V curve indicated that the on-set potential for mass transfer region was about $-0.6 \mathrm{~V}$ or even higher (Fig. S1). 
The on-set potential for EIS analysis at open circuit potential was all below $-0.6 \mathrm{~V}$ in this study. The impedance spectra consist of two different parts, the charge-transfer limited semicircle part at high frequencies and the Warburg diffusion-limited straight line part at low frequencies (Fig. S2). It illustrated that the total internal resistance of BES-S $\left(R_{\mathrm{S}}, 42.2 \Omega\right)$ was obviously lower than that of BES-V $\left(R_{\mathrm{V}}, 81.9 \Omega\right)$ and BES-H $\left(R_{\mathrm{H}}, 236.6 \Omega\right)$. Results further indicated that the BES-S had the advantage of low resistance, especially that the cathode resistance was only $8.7 \Omega$, which was much lower than that of the other two deployments ( $31.5 \Omega$ for vertical mode and $106.4 \Omega$ for horizontal mode) (Fig. 5C and Fig. S2). It was necessary for the confirmation that the improvement of electrodes deployments in single chamber BES reactor to be surrounding mode can efficiently improve the biocathode performance.

Detailed information on the internal resistance, such as the ohmic resistances $\left(R_{\mathrm{ohm}}\right)$, the charge transfer impedance $\left(R_{\mathrm{ct}}\right)$ and the mass transfer impedance $\left(R_{\text {diff }}\right)$, can be obtained on the analysis of anode, cathode and whole cell resistances to determine the reason why the total internal resistance was decreased. For $R_{\mathrm{ohm}}$ of the whole cell, $R_{\mathrm{ohm}}$ of BES-H showed a highest value of $57.8 \Omega$, which was much higher than that of BES-V (14.5 $\Omega$ ) and BES-S $(8.7 \Omega)$. It has demonstrated that the electrode spacing between the anode and cathode significantly influenced the $R_{\text {ohm }}$ (Zhang et al., 2013b). Obviously, the apparent differences in $R_{\text {ohm }}$ could be attributed to the different spacing between anode and cathode, especially the large area and small distance between anode and cathode in the surrounding mode. This would further affect the current for that a small $R_{\mathrm{ohm}}$ could give rise to a high current production, which explained the reason why different deployments had different current (Fig. 5B). Moreover, it should be pointed out that the different $R_{\mathrm{ohm}}$ also had a significant impact on the biofilm development during the startup of BES (Zhang et al., 2013b), which might be finally resulted in the rapid startup in the BES-S. For $R_{\mathrm{ct}}$ of the whole cell, the BES-S had the smallest $R_{\mathrm{ct}}(16.9 \Omega)$, while the BES$\mathrm{V}$ had much larger $R_{\mathrm{ct}}(51.7 \Omega)$ and the BES-H have the largest $R_{\mathrm{ct}}$ $(70.1 \Omega)$. These large resistances were probably due to the lack of suitable electron transfer pathways for operation. The $R_{\mathrm{ct}}$ can be a useful method to evaluate the stability of electrode biofilm. It has been reported that using a pre-acclimated cell suspension for inoculation could decrease the $R_{\mathrm{ct}}$ (Zhang et al., 2013a), thus the smallest $R_{\mathrm{ct}}$ in the BES-S might be also related to the rapid biofilm formation. According to EIS results, the decrease of whole cell $R_{\mathrm{ct}}$ in BES with surrounding electrode deployment was found to be contributed by both of $R_{\mathrm{ct}}$ decrease in anode and cathode, indicating the faster anodic and cathodic reactions compared to the other two configurations. As mentioned above, although an identical voltage of $0.3 \mathrm{~V}$ was applied in various designed BES, lower internal resistance in BES-S led to the higher current. This higher current was likely to cause the stronger stress to induce a higher electrochemical activity based on the electrons interaction between electrochemical active bacteria and the electrode, and resulted in the higher electrochemical reaction kinetics in BES-S. Considering many factors related to

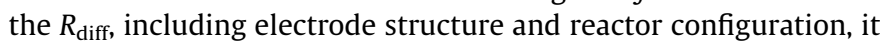
should design suitable structure and configuration to facilitate the rapid transport of the reactants, products, supporting electrolyte and substrate to reduce $R_{\text {diff }}$ (Zhao et al., 2009). EIS illustrated that the BES-S can effectively reduce the $R_{\text {diff, the performance would }}$ be improved further if the electrode modified.
Consequently, a suitable BES reactor structure with surrounding electrodes deployment played an important role in accelerating the BES start-up, reducing internal resistance $\left(R_{\mathrm{ohm}}, R_{\mathrm{ct}}\right.$ and $\left.R_{\mathrm{diff}}\right)$ and increasing the current, potentially becoming a necessary consideration for large-scale design.

\subsection{Decolorization performance with different electrodes deployments}

Decolorization performance with different electrodes deployments was also investigated. As the bioelectrochemical system was a complex system involving simultaneous biological and electrochemical processes, three different operating modes (BES, MFC and AnBR) were also compared to test the influence of bioelectrochemical stimulation on CR decolorization in BES with different electrodes deployments.

The CR-DE for $300 \mathrm{mg} \mathrm{L}^{-1} \mathrm{CR}$ was more than $90 \%$ within $23 \mathrm{~h}$ in all the tests. The CR reduction fitted first-order kinetic model well $\left(R^{2}>0.99\right)$. The $k$ shown in Table 3 indicated that the surrounding electrodes deployment not only won an advantage of decolorization in BES mode but also in MFC mode and anaerobic mode, followed by vertical and horizontal ones (Fig. 6A, B, C and Table 3). Moreover, the effect of applied voltage and biocathode on $\mathrm{CR}$ decolorization was more significant in surrounding deployment than vertical and horizontal ones. Results showed that the CR-DE of BES was 20\%, $17 \%$ and $11 \%$ higher than AnBR with surrounding, vertical and horizontal electrodes deployment, respectively, while the CR-DE in AnBR with the three electrodes deployments was almost the same $(71 \pm 1 \%$ ) (Fig. 6). Thus, the obvious different CR-DE was resulted from the BES mode. The better performance in BES-S might be attributed to the small resistance with surrounding deployment, for that a small resistance represented a low overpotential and a rapid reaction process (Zhao et al., 2009). This would further demonstrate the modified surrounding deployment in single chamber BES improved the protons and electrons transfer.

Thus, this study indicated that the surrounding deployment was not only suitable for the dual chamber BES as reported previously (Kong et al., 2013) but also suitable for the single chamber BES. It clearly proved that the compact structure with small distance and the surrounding deployment with large area between anode and cathode in the single chamber BES could decrease internal resistance (Fig. 5C), increase current (Fig. 5B) and finally accelerate the azo dye decolorization (Fig. 6).

The decolorization efficiency and electrochemical characterization results from the comparison of BES-H, BES-V and BES-S indicated that the surrounding deployment might be more potential for wastewater treatment in the single chamber BES in practice, such as domestic wastewater, brewery wastewater and hazardous substance mixed with co-substrate. This kind of wastewater was less toxicity to microorganisms in BES, probably leading to the obviously positive effects from combined anode and cathode. In addition, the aromatic amine products from azo dye reduction and decolorization could be thoroughly mineralized by the aerobic biological treatment (Cui et al., 2012). Such an aerobic bioreactor associated with efficient combined bioanode-biocathode BES with surrounding electrodes deployment would has a great potential for the azo dye-containing wastewater treatment.

Table 3

The rate constant $k$ of the three electrodes deployments under different operating conditions.

\begin{tabular}{|c|c|c|c|c|c|c|}
\hline Deployment & $k$-BES $\left(\mathrm{h}^{-1}\right)$ & $\mathrm{R}^{2}-\mathrm{BES}$ & $k$-MFC $\left(\mathrm{h}^{-1}\right)$ & $\mathrm{R}^{2}-\mathrm{MFC}$ & $k$-AnBR $\left(\mathrm{h}^{-1}\right)$ & $\mathrm{R}^{2}-\mathrm{AnBR}$ \\
\hline Horizontal & $0.176 \pm 0.011$ & 0.9977 & $0.149 \pm 0.015$ & 0.9968 & $0.099 \pm 0.005$ & 0.9957 \\
\hline Vertical & $0.292 \pm 0.024$ & 0.9947 & $0.199 \pm 0.022$ & 0.9974 & $0.127 \pm 0.007$ & 0.9949 \\
\hline Surrounding & $0.460 \pm 0.023$ & 0.9984 & $0.358 \pm 0.041$ & 0.9968 & $0.135 \pm 0.007$ & 0.9931 \\
\hline
\end{tabular}




\section{Conclusion}

This study demonstrated that the combined bioanode and biocathode can accelerate azo dye decolorization, and the microbical role in decolorization at biocathode played an important part. The comparison results with vertical electrodes deployment BES and horizontal electrodes deployment BES demonstrated that if modified the electrodes deployment to be surrounding deployment, the BES performance, including decolorization performance and electrochemical characterization, could be further improved. This study proved that the combined bioanode and biocathode with surrounding electrodes deployment was suitable for azo dye decolorization in the single chamber BES, potentially deriving a promising opportunity for wastewater treatment with modified electrodes deployment.

\section{Acknowledgements}

This research was supported by the National Natural Science Foundation of China (NSFC, Grant Nos. 51078100 and 31370157), National Science Foundation for Distinguished Young Scholars (Grant No. 51225802), Science Fund for Creative Research Groups of the National Natural Science Foundation of China (Grant No. 51121062), National High-tech R\&D Program of China (863 Program, Grant No. 2011AA060904), Ph.D. Programs Foundation of Ministry of Education of China (20102302110055) and Heilongjiang Science Foundation for Distinguished Young Scholars (Grant No. JC201003).

\section{Appendix A. Supplementary data}

Supplementary data associated with this article can be found, in the online version, at http://dx.doi.org/10.1016/ j.biortech.2013.10.027.

\section{References}

Aulenta, F., Tocca, L., Verdini, R., Reale, P., Majone, M., 2011. Dechlorination of trichloroethene in a continuous-Flow bioelectrochemical reactor: effect of cathode potential on rate, selectivity and electron transfer mechanisms. Environ. Sci. Technol. 45, 8444-8451.

Cao, Y., Hu, Y., Sun, J., Hou, B., 2010. Explore various co-substrates for simultaneous electricity generation and Congo red degradation in air-cathode single-chamber microbial fuel cell. Bioelectrochemistry 79, 71-76.

Cui, D., Kong, F., Liang, B., Cheng, H., Liu, D., Sun, Q., Wang, A., 2011. Decolorization of azo dyes in dual-chamber biocatalyzed electrolysis systems seeding with enriched inoculum. J. Environ. Anal. Toxicol. S3, 1-6.

Cui, D., Guo, Y., Cheng, H., Liang, B., Kong, F., Lee, H., Wang, A., 2012. Azo dye removal in a membrane-free up-flow biocatalyzed electrolysis reactor coupled with an aerobic bio-contact oxidation reactor. J. Hazard. Mater. 239-240, 257264.

Fernando, E., Keshavarz, T., Kyazze, G., 2012. Enhanced bio-decolourisation of acid orange 7 by Shewanella oneidensis through co-metabolism in a microbial fuel cell. Int. Biodeter. Biodegr. 72, 1-9.

Hou, B., Sun, J., Hu, Y., 2011a. Effect of enrichment procedures on performance and microbial diversity of microbial fuel cell for Congo red decolorization and electricity generation. Appl. Microbiol. Biotechnol. 90, 1563-1572.

Hou, B., Sun, J., Hu, Y., 2011b. Simultaneous Congo red decolorization and electricity generation in air-cathode single-chamber microbial fuel cell with different microfiltration, ultrafiltration and proton exchange membranes. Bioresour. Technol. 102, 4433-4438.

Hou, B., Hu, Y., Sun, J., 2012. Performance and microbial diversity of microbial fuel cells coupled with different cathode types during simultaneous azo dye decolorization and electricity generation. Bioresour. Technol. 111, 105-110.

Huang, L.P., Chai, X.L., Chen, G.H., Logan, B.E., 2011. Effect of set potential on hexavalent chromium reduction and electricity generation from biocathode microbial fuel cells. Environ. Sci. Technol. 45, 5025-5031.
Huang, L., Wang, Q., Quan, X., Liu, Y., Chen, G., 2013. Bioanodes/biocathodes formed at optimal potentials enhance subsequent pentachlorophenol degradation and power generation from microbial fuel cells. Bioelectrochemistry 94, 13-22.

Kong, F., Wang, A., Liang, B., Liu, W., Cheng, H., 2013. Improved azo dye decolorization in a modified sleeve-type bioelectrochemical system. Bioresour. Technol. 143, 669-673.

Li, Z., Zhang, X., Lin, J., Han, S., Lei, L., 2010. Azo dye treatment with simultaneous electricity production in an anaerobic-aerobic sequential reactor and microbial fuel cell coupled system. Bioresour. Technol. 101, 4440-4445.

Liang, B., Cheng, H., Kong, D., Gao, S., Sun, F., Cui, D., Kong, F., Zhou, A., Liu, W., Ren, N., Wu, W., Wang, A., Lee, D., 2013. Accelerated reduction of chlorinated nitroaromatic antibiotic chloramphenicol by biocathode. Environ. Sci. Technol. 47, 5353-5361.

Liu, H., Cheng, S., Huang, L.P., Logan, B.E., 2008. Scale-up of membrane-free singlechamber microbial fuel cells. J. Power Sources 179, 274-279.

Lohner, S.T., Becker, D., Mangold, K.M., Tiehm, A., 2011. Sequential reductive and oxidative biodegradation of chloroethenes stimulated in a coupled bioelectroprocess. Environ. Sci. Technol. 45, 6491-6497.

Luo, Y., Zhang, R., Liu, G., Li, J., Oin, B., Li, M., Chen, S., 2011. Simultaneous degradation of refractory contaminants in both the anode and cathode chambers of the microbial fuel cell. Bioresour. Technol. 102, 3827-3832.

Mu, Y., Rabaey, K., Rozendal, R.A., Yuan, Z., Keller, J.R., 2009. Decolorization of azo dyes in bioelectrochemical systems. Environ. Sci. Technol. 43, 5137-5143.

Savizi, I.S.P., Kariminia, H.R., Bakhshian, S., 2012. Simultaneous decolorization and bioelectricity generation in a dual chamber microbial fuel cell using electropolymerized-enzymatic cathode. Environ. Sci. Technol. 46, 6584-6593.

Solanki, K., Subramanian, S., Basu, S., 2013. Microbial fuel cells for azo dye treatment with electricity generation: a review. Bioresour. Technol. 131, 564-571.

Sun, J., Bi, Z., Hou, B., Cao, Y., Hu, Y., 2011a. Further treatment of decolorization liquid of azo dye coupled with increased power production using microbial fuel cell equipped with an aerobic biocathode. Water Res. 45, 283-291.

Sun, J., Hu, Y., Hou, B., 2011b. Electrochemical characterization of the bioanode during simultaneous azo dye decolorization and bioelectricity generation in an air-cathode single chambered microbial fuel cell. Electrochim. Acta 56, 68746879.

Sun, J., Li, Y.M., Hu, Y.Y., Hou, B., Xu, Q., Zhang, Y.P., Li, S.Z., 2012. Enlargement of anode for enhanced simultaneous azo dye decolorization and power output in air-cathode microbial fuel cell. Biotechnol. Lett. 34, 2023-2029.

Sun, J., Li, Y.M., Hu, Y.Y., Hou, B., Zhang, Y.P., Li, S.Z., 2013. Understanding the degradation of Congo red and bacterial diversity in an air-cathode microbial fuel cell being evaluated for simultaneous azo dye removal from wastewater and bioelectricity generation. Appl. Microbiol. Biotechnol. 97, 3711-3719.

Wang, A., Cheng, H., Liang, B., Ren, N., Cui, D., Lin, N., Kim, B., Rabaey, K., 2011. Efficient reduction of nitrobenzene to aniline with a biocatalyzed cathode. Environ. Sci. Technol. 45, 10186-10193.

Wang, Y., Wang, A., Liu, W., Kong, D., Tan, W., Liu, C., 2013a. Accelerated azo dye removal by biocathode formation in single-chamber biocatalyzed electrolysis systems. Bioresour. Technol. 146, 740-743.

Wang, Y., Wang, A., Liu, W., Sun, Q., 2013b. Enhanced azo dye removal through anode biofilm acclimation to toxicity in single-chamber biocatalyzed electrolysis system. Bioresour. Technol. 142, 688-692.

Wen, Q., Wu, Y., Zhao, L., Sun, Q., Kong, F., 2010. Electricity generation and brewery wastewater treatment from sequential anode-cathode microbial fuel cell. J. Zhejiang Univ. Sci. B 11, 87-93.

Xafenias, N., Zhang, Y., Banks, C.J., 2013. Enhanced performance of hexavalent chromium reducing cathodes in the presence of Shewanella oneidensis MR-1 and lactate. Environ. Sci. Technol. 47, 4512-4520.

Xing, D.F., Cheng, S.A., Regan, J.M., Logan, B.E., 2009. Change in microbial communities in acetate- and glucose-fed microbial fuel cells in the presence of light. Biosens. Bioelectron. 25, 105-111.

Xu, Q., Sun, J., Hu, Y., Chen, J., Li, W.-J., 2013. Characterization and interactions of anodic isolates in microbial fuel cells explored for simultaneous electricity generation and Congo red decolorization. Bioresour. Technol. 142, 101-108.

Zhang, F., Xia, X., Luo, Y., Sun, D., Call, D.F., Logan, B.E., 2013a. Improving startup performance with carbon mesh anodes in separator electrode assembly microbial fuel cells. Bioresour. Technol. 133, 74-81.

Zhang, L., Li, J., Zhu, X., Ye, D., Liao, Q., 2013b. Anodic current distribution in a literscale microbial fuel cell with electrode arrays. Chem. Eng. J. 223, 623-631.

Zhang, X., Epifanio, M., Marsili, E., 2013c. Electrochemical characteristics of Shewanella loihica on carbon nanotubes-modified graphite surfaces. Electrochim. Acta 102, 252-258.

Zhao, F., Slade, R.C.T., Varcoe, J.R., 2009. Techniques for the study and development of microbial fuel cells: an electrochemical perspective. Chem. Soc. Rev. 38, 1926-1939.

Zhu, X., Zhang, L., Li, J., Liao, Q. Ye, D., 2013. Performance of liter-scale microbial fuel cells with electrode arrays: effect of array pattern. Int. J. Hydrogen Energy. http://dx.doi.org/10.1016/j.ijhydene.2013.06.052. 